\title{
A robotic guide for blind people Part 2: Gender and national analysis of a multi-national survey and the application of the survey results and the CAT model to framing robot design specifications
}

\author{
Marion A. Hersh ${ }^{\mathrm{a}, *}$ and Michael A. Johnson ${ }^{\mathrm{b}}$ \\ ${ }^{a}$ Biomedical Engineering, University of Glasgow, Glasgow, Scotland \\ ${ }^{\mathrm{b}}$ University of Strathclyde, Glasgow, Scotland
}

\begin{abstract}
This paper presents a gender and country-based analysis of the results of a multi-national survey questionnaire on the attitudes, requirements and preferences of blind and visually impaired people for a robotic guide. This is introduced by a brief summary of the findings of the survey introduced and reported in Part 1 and a brief overview of some of the technologies that are currently used in the construction of mobile robotic guides. An analysis of the gender dimension revealed very few differences in preferences or requirements between male and female respondents. There was also considerable commonality of preferences and requirements across the four countries, France, Italy, Spain and the UK, for which the comparative analysis was carried out. This implies that, at least initially, one robotic guide can be developed to be used in a number of different countries and by both women and men. The survey results were then applied to develop design specifications for a mobile robotic guide for blind and visually impaired people. The framework of the Comprehensive Assistive Technology (CAT) model developed by the authors was used to organise the information and structure the development of the design specifications. Further work will involve the construction of design mock-ups to implement the design specifications and their investigation with end-users to choose a design for further development.
\end{abstract}

Keywords: Design, robotic guide, gender and national analysis, survey, end-user involvement, Comprehensive Assistive Technology model, design specifications

\section{Introduction}

Part 1 of the paper [15] presented the methodology and discussed some of the results of a survey to investigate the attitudes to and requirements and

\footnotetext{
*Corresponding author: Marion A. Hersh, Biomedical Engineering, University of Glasgow, Glasgow G12 8LT, Scotland. Tel.: +44 141330 4906; Fax: +44 141330 6004; E-mail: marion.hersh@ glasgow.ac.uk.
}

preferences of blind and visually impaired people for a robotic guide. As indicated in Part 1 of the paper, this research was carried out largely in response to the fact that, despite the development of a number of travel aids, most of them have not gone beyond the prototype stage and the long cane and guide dog are still the most popular obstacle avoidance devices. There has been some progress in the area of navigation and wayfinding with the development of global positioning systems (GPS) and environmental information 
beacons. However, GPS has the disadvantage of lack of precision and only relatively small numbers of environmental information beacons have been installed to-date and they are limited to a small number of countries. Furthermore, the different systems are not currently mutually compatible and each requires its own receiver or reader [11].

This Part 2 paper presents the gender and country analysis of the results and draws on the results in both parts of the paper to develop design specifications for a robotic guide. The gender and country analysis is important for a number of reasons. As indicated in Part 1, there has been very little work on end-user attitudes to robotic guides and their requirements and preferences for particular features. However, potential end-users have a number of different characteristics and it is therefore important to investigate whether, and, if so, in what ways users' attitudes, requirements and preferences depend on user characteristics. This will inform the design and, in particular, determine whether one design, customisation options or different designs for different groups of end-users will be required. Due to space limitations, a decision was made to present a detailed dimension of the gender and country analysis of end-user attitudes, requirements and preferences in this paper, with a more limited analysis of the results based on age and degree of visual impairment in the Part 1 paper. Development of this analysis in more detail, comparable to the analysis for gender and country in this paper, would require additional data for end-users in the $71+$ age range. The results reported here are one of very few multi-lingual multi-country studies in the area of assistive technology and therefore may have implications for developments in the area of methodology.

The overall, gender and country results on enduser attitudes, requirements and preferences for robotic guide features presented in the two parts of the paper are further used in this paper to develop design specifications for a robotic guide. The Comprehensive Assistive Technology (CAT) model developed by the authors $[13,14]$ is used to structure the end-user information and the discussion of the relationship between design specifications and end-user requirements and preferences.

The presentation of the gender and country results and the development of design specifications are introduced and supported by a brief overview of some of the technologies used in existing mobile robots in Section 2. This is followed by a discussion of the gender and country dimension of the questionnaire results in Section 3 and the application of the survey results and the CAT model to the design of a robotic guide in Section 4. Discussion and conclusions are presented in Section 5. To support the application in Section 4, a brief summary of the questionnaire results presented in Part 1 is given in Section 1.1.

\subsection{Brief summary of the results of the questionnaire on attitudes to, requirements and preferences for a robotic guide}

The survey obtained 80 useful responses with respondents having sufficiently varied characteristics to give reasonable coverage of the end-user community. The overwhelming majority of respondents used a mobility device, generally a long cane, and from their self-reports generally achieved successful mobility with it.

Of the respondents who did not use a mobility device, about three quarters felt they could benefit from using one at least sometimes. Slightly fewer than half the respondents used a navigation device en route, most commonly a global positioning system (GPS), and most respondents were confident about reaching their destination with it. About $90 \%$ of the respondents were interested in the robotic guide providing information on the current location, reading street names, obstacle avoidance and navigation, with three quarters wanting the facility to locate goods.

A number of additional functions were suggested, frequently by several respondents. These included information about the location and points of interests; information about the status of traffic lights and support in finding pedestrian crossings, as well as the ability to contact the emergency services or the user's family and give them the user's location. There were also numerous suggestions for supporting the use of public transport, as well as suggestions of specific functions in the areas of navigation (and wayfinding), reading information and obstacle avoidance.

Respondents considered the robot's appearance to be very important, but were not particularly enthusiastic about any of the suggested designs. However, they almost universally wanted a robot that was discreet and inconspicuous and resembled an object in every-day use, as well as small, lightweight, easily portable and attractive. Some respondents wanted a pocket-sized device, whereas others would be satisfied with a device that could fit on or under a table. Respondents were also 
concerned about the robot having a long battery life, with suggestions of 16 hours and several days, being robust, lasting a long time, not requiring maintenance and being easy and intuitive to use, as well as to clean. Respondents wanted to use the robot in a variety of different circumstances, including everyday use and for going to new destinations.

Speech was the preferred method for communicating with the robot, followed by a keypad, then a joystick or switch for input. There was little interest in Braille for output and some respondents wanted a customisable device, with a range of options, including vibro-tactile feedback.

\section{Overview of some of the technologies used in existing mobile robots}

The final aim of this paper is to use the responses to the end-user survey together with the Comprehensive Assistive Technology model to derive preliminary design specifications for a mobile robotic guide for blind and visually impaired people. To support the development of a guide which takes account of endusers' preferences, and requirements, as well as what is currently feasible technologically, a brief review of some of the technologies used in existing mobile robots will now be presented.

\subsection{Localisation, navigation and obstacle and collision avoidance}

Localisation to determine the robot's pose or $\mathrm{x}-\mathrm{y}$ coordinates and heading direction is an essential precondition for navigation. It also supports the avoidance of obstacles, such as downward escalators, that robots may be unable to perceive with their sensors. Three types of robot localisation problem, all of which are relevant to robotic guides for blind people, are discussed in the literature [26]:

(i) updating the robot's position from an initially known pose to compensate for small odometry errors as the robot moves;

(ii) localisation from an initially unknown pose;

(iii) localisation when the robot disappears from a known to a random pose without being informed, simulating the robot's ability to recover from a catastrophic failure of the localisation routine.
However, most of the discussion in the literature has been of robot localisation in bounded, structured indoor environments and there has been very little discussion of robot localisation in unbounded and sometimes less structured outdoor environments. Localisation is generally carried out by finding the best possible match between the local map of current observations and the global map. The two main approaches to modelling indoor robot environments are grid based $[6,22]$ and topological $[19,20]$ and these approaches have been combined to benefit from their complementary advantages and, as much as possible, cancel out their complementary disadvantages [27].

Mobile robots use some combination of laser range finders, ultrasonar and infrared sensors and (video) cameras to detect obstacles, with some robots having several different sensor systems and others only one. Lasers are considered the most accurate, but are expensive. Mobile robots may also use data from a map supplied or constructed using sensor data to detect obstacles which are not easy to detect using its sensory systems. When an obstacle is detected, the data is processed and the information transmitted to the robot wheels or steering motors to change trajectory to avoid the obstacle.

The obstacle and collision avoidance algorithms used include the $(\mu)$ dynamic window algorithm [3, 7], which integrates sensor and map data and the (minimal) vector field histogram $[1,25]$.

\subsection{Locomotion, the end-user interface and control strategies}

Most mobile robots are wheeled. This gives them advantages over legged robots with regards to stability, but disadvantages in terms of the inability to go up and down stairs, move on rough terrain, move sideways or turn quickly. However, the pre-prototype robotic wheelchair developed by the OMNI project [2] uses mecanum wheels [5] to give sideways and rotational, as well as forward motion and the iBOT 3000 mobility system [28] is able to go up and down stairs.

The design of legged robots is a more complex problem than the design of wheeled robots. In the case of robotic guides, this involves the design of a smooth gait at walking pace which does not look ridiculous. In addition, the robot should be stable, not overbalance the user and be able to adjust its pace to that of the user. Several different robots with two, four or six legs have been developed, for instance $[9,16,17,29,30]$. 
Some of these robots have mechanisms to prevent overbalance, to adapt to new terrain and/or to learn new walking patterns.

Mobile robotic guides and other mobile robots use a combination of speech and haptics to communicate information to end-users. In many cases, the path to follow is communicated haptically by the user feeling the movement of the device. Robotic tour guides also use 'facial expressions' and sometimes on screen announcements. User input is generally tactile, including the use of Braille, push buttons, a numeric keypad, a mini joystick and control switches. However, in the case of some robotic tour guides [3, 4], this involves coloured switches and a touch screen and is consequently inaccessible to blind people.

Robotic wheelchairs generally allow a range of different types of input devices, including switches operated by different parts of the body, joysticks and laser pointers. Particular care and a degree of imagination would be required in choosing suitable input (devices) for blind people who are also users of motorised wheelchairs, particularly if hand-operated input devices are not suitable for them.

The movement of wheeled mobile robots is generally achieved through control of the motors which drive the wheels, either together or independently of each other. The computer that calculates the controller may be integrated into the robot or off-board, possibly in a laptop [8]. Otherwise, the use of off-board computers will severely restrict the range of motion of the robot. Control strategies can be based on coupled or decoupled models of the wheel motion and may control the robot's translational and rotational velocities together or separately. Many mobile robot controllers have a hierarchical structure of levels of increasing complexity $[23,24]$. In the case of legged robots, the movement of the leg joints is controlled. In some case different control strategies are used to control different aspects of the motion, with the coupling effects between them treated as perturbations [10].
As is the case with robotic wheelchairs and other devices which have a close interaction with the user and are in principle able to control some activities directly, there is the issue of 'shared' control between the user and the robot. There is a range of options from all decisions being made by the user and communicated to the robot to a totally autonomous robot. Normally the preferred balance is one in which the user makes high level decisions e.g. on the destination and the robot takes decisions to reduce stress on the user without reducing their autonomy e.g. on obstacle avoidance actions. However, some users may prefer to be consulted before the robot takes any action. There are also issues of potential deadlock and safety critical situations. Therefore the robot should have a shared control module, as well as the option for the user to determine the balance of shared control between themselves and the robot.

The literature review in Part 1 of this paper showed that progress in developing mobile robotic guides for blind and visually impaired people has been limited. In addition to the problems of mechanical design, mobile robotic guides have complex control problems (see Fig. 1), including shared control, navigation, processing of sensor data, obstacle avoidance and delivering reference signals to the drive units. To date development work on this highly interactive and multivariable control problem has been limited.

\section{Analysis by gender and country of the survey results}

For the survey previously reported in Part 1 a gender and national analysis is given in Sections 3.1 and 3.2 , respectively. Where statistical significance is discussed without further qualifiers, this is always at the 0.05 level. However, in a few cases the $p$ values indicate that significance also holds at the 0.01 level. The

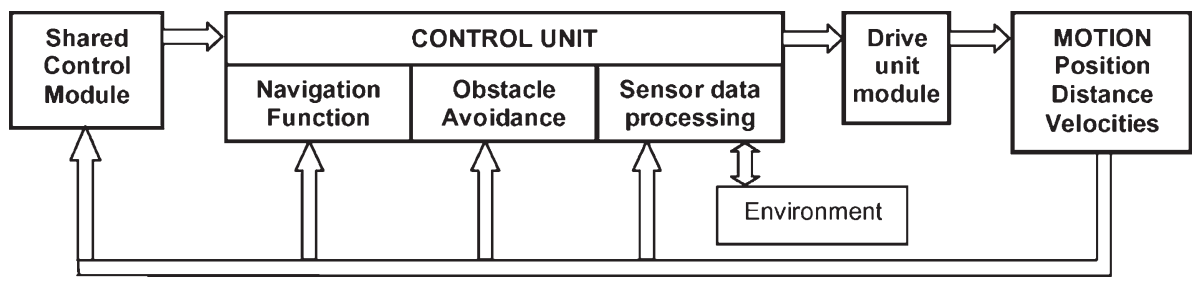

Fig. 1. Control structure of a mobile robotic guide. 
Table 1

Country, age and visual status profile of male and female respondents

\begin{tabular}{|c|c|c|c|c|c|c|c|c|c|c|c|c|c|}
\hline & \multirow[t]{2}{*}{ Sample size } & \multicolumn{5}{|c|}{ Country $(\%)$} & \multicolumn{5}{|c|}{ Age profile $(\%)$} & \multicolumn{2}{|c|}{ Visual status $(\%)$} \\
\hline & & France & Italy & Poland & UK & Other & $16-25$ & $26-40$ & $41-60$ & $61-70$ & $71+$ & VI & Blind \\
\hline Female & 32 & 12.5 & 25.0 & 37.5 & 12.5 & 12.5 & 21.9 & 46.9 & 18.8 & 12.5 & 0 & 0.25 & 71.9 \\
\hline Male & 48 & 18.8 & 27.1 & 25.0 & 22.9 & 6.3 & 4.2 & 31.3 & 50.0 & 12.5 & 2.1 & 31.3 & 68.8 \\
\hline
\end{tabular}

Fisher exact test with software developed by Langsrud (undated) was used to determine statistical significance in the case of the data on functions (question $\mathrm{C} 1$ ) and communication with the robot (questions C5a and C5c). A contingency table $\chi^{2}$ test with five degrees of freedom was used for the data on design (question C2), appearance (question $\mathrm{C} 3 \mathrm{a}$ ) and circumstances (question C4) with software developed by [18]. In cases where the presence of zeros across a row would have prevented the use of a test with five degrees of freedom, data has been combined and a test with four degrees of freedom used.

\subsection{Gender analysis of the survey results}

There were significantly more male than female respondents. Further research involving a larger sample would be required to determine whether this is coincidental or indicates greater interest by men than women in a robotic guide. The tables in this section present an analysis of the data by gender and include average values over all respondents of both sexes for comparison. Where $p$-values are given these are for the differences between male and female respondents. Table 1 presents the data on male and female respondents by country, age and visual status.

Respondents from Belgium, Canada, Spain and the USA have been grouped together under 'other' since the numbers involved were small. From Table 2, women and men were both strongly interested in all the functions suggested and the percentage of women and men interested in particular functions were broadly similar. A statistically significant difference between the preferences of men and women was found only in the case of navigation $(p=0.002)$. In the case of reading street names, the values were very close together and the percentage for women interested was very slightly higher than that of men. However, the percentage of men interested in each of the other functions was greater than that of women. The average level of interest over all the functions was $81.3 \%$ for women compared with $90.4 \%$ for men and this difference is
Table 2

Gender analysis of the percentages of respondents interested in particular functions

\begin{tabular}{lcccl}
\hline C1 function & Female & Male & All & $p$-value \\
\hline Number of respondents & 32 & 48 & 80 & \\
Obstacle avoidance & $84.4 \%$ & $91.7 \%$ & $88.8 \%$ & 0.26 \\
Informing user of location & $87.5 \%$ & $93.8 \%$ & $91.3 \%$ & 0.28 \\
Navigation & $75.0 \%$ & $97.9 \%$ & $88.8 \%$ & 0.0022 \\
Location of goods & $68.8 \%$ & $79.2 \%$ & $75.0 \%$ & 0.21 \\
Reading street names & $90.6 \%$ & $89.6 \%$ & $90.0 \%$ & 0.60 \\
Average over functions & $81.3 \%$ & $90.4 \%$ & $86.8 \%$ & 0.007 \\
Other & $34.4 \%$ & $43.8 \%$ & $40.0 \%$ & 0.27 \\
\hline
\end{tabular}

Table 3

Gender analysis of the grading of different robot designs and the importance of robot appearance

\begin{tabular}{lccc}
\hline C2 and C3a design and & Female & Male & All \\
appearance & 31 & 47 & 78 \\
\hline C2 number of respondents & 2.2 & 2.0 & 2.1 \\
Robotic dog & 2.4 & 1.9 & 2.1 \\
Humanoid & 2.2 & 2.8 & 2.5 \\
Robot & 2.2 & 2.2 & 2.2 \\
Mobility scooter & 2.1 & 1.9 & 2.0 \\
Robotic scooter & 1.7 & 1.6 & 1.4 \\
Frame with seat+ & 1.4 & 1.2 & 1.3 \\
Frame & 2.1 & 2.3 & 2.2 \\
Robotic shopper & 0.7 & 0.9 & 0.8 \\
Lawnmower & 1.8 & 1.8 & 1.8 \\
Base with cane & 1.9 & 1.9 & 1.8 \\
Average over these designs & 31 & 46 & 77 \\
C3a number of respondents & 3.9 & 3.7 & 3.8 \\
Importance of appearance & & & \\
\hline
\end{tabular}

statistically significant $(p=0.007)$. This indicates that there may be a difference in the number of functions men and women would like the robot to have, with women wanting fewer functions than men.

From Table 3 , the differences in preferences for most of the design options for the robot were very small. However, it was interesting to note the slightly greater differences between the preferences for an obvious robot and a robot which looks like a person, with men preferring the first and women the second. Although these differences were not statistically 
significant ( $p=0.83$ for humanoid and 0.32 for obvious robot), it would be interesting to investigate them with a larger sample. Women attached slightly greater importance to the robot's appearance than men did, but the difference was not statistically significant $(p=0.69)$.

From Table 4, both men and women were enthusiastic about using the robot in all the circumstances suggested. In most cases, the differences between their preferences were very small and in some cases the values were identical, as was the average value over all the circumstances. There were slightly greater differences in the case of supermarkets, which women considered more important and everyday use, where men had the greater preference, but the differences were not statistically significant for this sample size ( $p=0.21$ for supermarkets and 0.39 for everyday use).

Table 5 shows that preferences for the different methods suggested for giving instructions and receiving information from the robot were broadly similar for men and women, though more men preferred using speech to give information and more women preferred receiving information through speech. In the

Table 4

Gender analysis of the grading of the usefulness of the robot in different circumstances

\begin{tabular}{lccc}
\hline C4 circumstances & Female & Male & All \\
\hline Number of respondents & 31 & 46 & 77 \\
Supermarket & 4.6 & 4.2 & 4.4 \\
Airport & 4.3 & 4.2 & 4.2 \\
Railway station & 4.6 & 4.6 & 4.6 \\
Public buildings & 4.1 & 4.1 & 4.1 \\
Everyday use & 3.8 & 4.2 & 4.0 \\
New destinations & 4.7 & 4.9 & 4.8 \\
Average over circumstances & 4.4 & 4.4 & 4.3 \\
Other & 0.1 & 0.4 & 0.3 \\
\hline
\end{tabular}

Table 5

Gender analysis of the percentage of respondents who would use particular types of input and output

\begin{tabular}{lclll}
\hline & Female & Male & All & $p$ value \\
\hline C5a give instructions & & & & \\
Number of respondents & 32 & 48 & 80 & \\
Speech & $62.5 \%$ & $70.8 \%$ & $67.5 \%$ & 0.29 \\
Keypad & 37.5 & $43.8 \%$ & $41.3 \%$ & 0.37 \\
Joystick or switch & $31.3 \%$ & $25.0 \%$ & $27.5 \%$ & 0.35 \\
Other & $6.3 \%$ & $14.6 \%$ & $11.3 \%$ & 0.21 \\
C5c receive information & & & & \\
Number of respondents & 30 & 48 & 78 & \\
Speech & $90.0 \%$ & $85.4 \%$ & $87.2 \%$ & 0.41 \\
Braille & $20.0 \%$ & $10.4 \%$ & $14.1 \%$ & 0.42 \\
Other & $13.3 \%$ & $25.0 \%$ & $20.5 \%$ & 0.17 \\
\hline
\end{tabular}

case of both men and women, a higher percentage of respondents wanted to receive information than give instructions using speech. The difference was statistically significant for women $(p=0.011)$, but not for men $(p=0.067)$. The ordering of preferences for giving instructions was speech, keypad, joystick or switch for both men and women. About twice as many women as men wanted to receive Braille output, though the difference was not statistically significant. A larger sample would be required to investigate this further. However, it is possible that a higher percentage of blind and visually impaired women than men are able to read Braille, but statistics do not seem to be available.

\subsection{Analysis by country of the survey results}

The tables in this section present the results organised by country for France, Italy, Poland and the UK. Where appropriate, they include an average over all the items in the table e.g. interest in particular functions for each country. Data for Belgium, Canada, Spain and the USA has not been included due to the very small number of respondents from each of these countries. The data for England, Northern Ireland and Scotland has been grouped together under the UK for the same reason. Table 6 shows the gender, age and visual status profiles for respondents in France, Italy, Poland and the UK.

Table 7 shows that broadly similar high percentages of respondents were interested in the obstacle avoidance and location functions in the four countries. The highest value was obtained in the UK, where all respondents were interested in these functions, with slightly lower figures in the other countries. All the respondents were interested in navigation in Poland and the UK, about $90 \%$ in France and only $70 \%$ in Italy. There were statistically significant differences in the levels of interest in Italy and Poland $(p=0.007)$ and Italy and the UK $(p=0.03)$, but not Italy and France $(p=0.15)$. A much higher percentage of respondents in Poland was interested in locating goods than in the other three countries, with the differences between Poland and both Italy $(p=0.005)$ and France $(p=0.01)$, but not the UK $(p=0.06)$ statistically significant. Interest in reading street names was much greater in Poland and Italy than in France and the UK, but this difference was not statistically significant (with the smallest value of $p=0.15$ for Poland and the UK). Over four fifths of the respondents in each country would like all the functions, with the highest percentage of $95 \%$ in Poland 
Table 6

Gender, age and visual status profile of respondents in different countries

\begin{tabular}{|c|c|c|c|c|c|c|c|c|c|c|}
\hline & \multirow[t]{2}{*}{ Sample size } & \multicolumn{2}{|c|}{ Gender $(\%)$} & \multicolumn{5}{|c|}{ Age profile $(\%)$} & \multicolumn{2}{|c|}{ Visual status (\%) } \\
\hline & & M & $\mathrm{F}$ & $16-25$ & $26-40$ & $41-60$ & $61-70$ & $71+$ & VI & Blind \\
\hline France & 13 & 69 & 31 & 0 & 31 & 62 & 8 & 0 & 15 & 85 \\
\hline Italy & 21 & 62 & 38 & 29 & 43 & 19 & 10 & 0 & 20 & 80 \\
\hline Poland & 24 & 50 & 50 & 8 & 50 & 25 & 13 & 4 & 31 & 69 \\
\hline UK & 15 & 73 & 27 & 7 & 20 & 47 & 27 & 0 & 53 & 47 \\
\hline
\end{tabular}

Table 7

National analysis of the percentages of respondents interested in particular functions

\begin{tabular}{lllccc}
\hline C1 functions & France & Italy & Poland & UK & $\begin{array}{c}\text { Four } \\
\text { countries }\end{array}$ \\
\hline Number of respondents & 13 & 21 & 24 & 15 & 73 \\
Obstacle avoidance & $92.3 \%$ & $85.7 \%$ & $91.7 \%$ & $100.0 \%$ & $91.5 \%$ \\
Location & $92.3 \%$ & $90.5 \%$ & $91.7 \%$ & $100.0 \%$ & $92.9 \%$ \\
Navigation & $92.3 \%$ & $71.4 \%$ & $100.0 \%$ & $100.0 \%$ & $90.1 \%$ \\
Location of goods & $61.5 \%$ & $61.9 \%$ & $95.8 \%$ & $73.3 \%$ & $76.1 \%$ \\
Read street names & $84.6 \%$ & $95.2 \%$ & $95.8 \%$ & $80.0 \%$ & $91.6 \%$ \\
Average & $84.6 \%$ & $81.0 \%$ & $95.0 \%$ & $90.7 \%$ & $86.8 \%$ \\
\hline
\end{tabular}

and the lowest of $81.0 \%$ in Italy, but the difference was not statistically significant $(p=0.11)$.

Table 8 shows that respondents in all four countries considered the appearance of the robot very important, with the lowest value of 3.5 in Italy and the highest of 4.0 in the UK. This difference is not statistically significant $(p=0.27)$ and the fact that all the countries give a high value to appearance is more important than the differences between them. However, there are some differences in the preferences across countries for the different designs. Respondents in France were fairly

Table 8

National analysis of the average grading of different robot designs and the importance of robot appearance

\begin{tabular}{lccccc}
\hline $\begin{array}{l}\text { C2 and C3a design } \\
\text { and appearance }\end{array}$ & France & Italy & Poland & UK & $\begin{array}{c}\text { Four } \\
\text { countries }\end{array}$ \\
\hline Number of respondents & 13 & 19 & 24 & 15 & 71 \\
Robotic dog & 1.8 & 1.8 & 3.0 & 1.6 & 2.3 \\
Humanoid & 1.9 & 2.1 & 2.7 & 2.2 & 2.2 \\
Robot & 3.5 & 2.5 & 2.5 & 1.1 & 2.6 \\
Mobility scooter & 2.1 & 3.0 & 2.1 & 1.8 & 2.1 \\
Robotic scooter & 1.7 & 2.4 & 2.0 & 1.7 & 2.0 \\
Frame with seat+ & 0.6 & 1.3 & 2.3 & 1.1 & 1.6 \\
Frame & 0.8 & 1.2 & 1.6 & 1.9 & 1.3 \\
Robotic shopper & 2.6 & 1.9 & 2.8 & 1.2 & 2.3 \\
Lawnmower & 0.8 & 0.6 & 0.5 & 2.2 & 0.8 \\
Base with cane & 2.8 & 2.0 & 1.0 & 0.0 & 1.8 \\
Average over all designs & 1.7 & 2.0 & 2.0 & 1.6 & 1.8 \\
Number of respondents & 11 & 20 & 24 & 15 & 70 \\
Importance of appearance & 3.9 & 3.5 & 3.9 & 4.0 & 3.8 \\
\hline
\end{tabular}

enthusiastic about the obvious robot which they evaluated at 3.5, the highest value given to any of the designs in any of the countries, followed by fairly positive feelings for the robotic base with cane attached (2.8) and the robotic shopper (2.6). Respondents in Italy gave their highest value (3.0) to the mobility scooter, followed by the obvious robot (2.5) and robotic scooter (2.4). Respondents in Poland were most positive about the robot dog (3.0), followed by the robotic shopper (2.8) and humanoid robot (2.7). Respondents in the UK preferred the robotic shopper (2.8) followed by the lawnmower (2.2) and humanoid robot (2.2). However, respondents in the other three countries rated the lawnmower very poorly $(0.5,0.6$ and 0.8$)$. Feelings about the base with cane attached were also very mixed, with it rated at 0 by the respondents in the UK and 2.8 by the French respondents. On average, lower ratings were given by respondents in the UK (1.6) and France (1.7) than in Poland and Italy (both 2.0).

Table 9 shows that respondents in all four countries considered the robot would be very useful in all the suggested circumstances. There was very little difference between the average values for the different countries. This indicates that, while there were slight differences in the perceived usefulness of the different functions in the different countries, there were no real differences across countries in the extent and range of functions that respondents would like to have from the robot. The greatest difference in perceived usefulness was for everyday use, with the lowest value of 3.7 in Italy 
Table 9

National analysis of the average grading of the robot in particular circumstances

\begin{tabular}{lccccc}
\hline C4 circumstances & France & Italy & Poland & UK & $\begin{array}{c}\text { Four } \\
\text { countries }\end{array}$ \\
\hline Number of respondents & 13 & 20 & 24 & 14 & 72 \\
Supermarket & 4.5 & 4.2 & 4.3 & 4.6 & 4.4 \\
Airport & 4.2 & 4.2 & 4.1 & 4.6 & 4.2 \\
Railway station & 4.8 & 4.6 & 4.5 & 4.6 & 4.6 \\
Public buildings & 4.1 & 4.2 & 3.9 & 4.2 & 4.1 \\
Everyday use & 4.2 & 3.7 & 3.9 & 4.5 & 4.0 \\
New destinations & 4.7 & 4.7 & 4.9 & 4.9 & 4.8 \\
Average & 4.4 & 4.2 & 4.3 & 4.3 & 4.4 \\
\hline
\end{tabular}

and the highest value of 4.5 in the UK, but the difference was not statistically significant $(p=0.7)$ and a larger sample would be required to investigate this difference further.

From Table 10, speech was the preferred mode of providing instructions and receiving information from the robot in the four countries, with a higher percentage of respondents in each country interested in using speech to receive information than to give instructions. There were similar levels of interest in speech input in Poland, France and the UK, whereas the level of interest in speech output was similar in Poland and France, but considerably lower in the UK. Interest in both speech input and output was less in Italy than in the other three countries. In all four countries there was greater interest in speech output than speech input. The ratio of respondents interested in speech input to output was comparable at about two thirds in France, Italy and Poland, but considerably greater at about $90 \%$ in the UK.

In all four countries, the ranking of interest for input devices was speech, keypad, joystick or switch. However, there were significant differences in the percentages of respondents interested in keypad, and joystick or switch in the different countries and there were no obvious patterns. For instance, equal numbers of respondents in Poland were interested in both a keypad, or joystick or switch, at slightly under a third in both cases, whereas ten times as many respondents in Italy were interested in a keypad as in a joystick or switch, with nearly half interested in a keypad. Interest in Braille output varied from $0 \%$ in the UK to $38.5 \%$ in France.

\subsection{Brief summary of gender and country-based analysis}

Both men and women and respondents in all four countries were very strongly interested in the functions of obstacle avoidance, being informed of their location and reading street names. Men and respondents in Poland, the UK and France were also very strongly interested in navigation (92-100\%), whereas respondents in Italy and women were somewhat less interested, though interest was still at the $70-75 \%$ level. Respondents in Poland were very strongly interested in locating goods, whereas interest was considerably lower in France and Italy at about $60 \%$ and in the UK at about $70 \%$. However, it could not be said that

Table 10

National analysis of the percentage of respondents who would use particular types of input and output

\begin{tabular}{lccccc}
\hline & France & Italy & Poland & UK & Four countries \\
\hline C5a give instructions & & & & & \\
Number of respondents & 13 & 21 & 24 & 15 & 73 \\
Speech & $62.5 \%$ & $52.4 \%$ & $66.7 \%$ & $66.7 \%$ & $68.5 \%$ \\
Keypad & 37.5 & $47.6 \%$ & $29.2 \%$ & $46.7 \%$ & $39.7 \%$ \\
Joystick or switch & $31.3 \%$ & $4.8 \%$ & $29.2 \%$ & $20.0 \%$ & $27.4 \%$ \\
Other & $6.3 \%$ & $4.8 \%$ & $12.5 \%$ & $13.3 \%$ & $11.0 \%$ \\
C5c receive information & & & & & \\
Number of respondents & 13 & 20 & 24 & 14 & 71 \\
Speech & $100 \%$ & $70.0 \%$ & $95.8 \%$ & $78.6 \%$ & $85.9 \%$ \\
Braille & $38.5 \%$ & $20.0 \%$ & $16.7 \%$ & $0 \%$ & $14.1 \%$ \\
Other & $69.2 \%$ & $15.0 \%$ & $8.3 \%$ & $57.1 \%$ & $21.1 \%$ \\
Ratio of preferences for speech & & & & & \\
$\quad$ for input to output & & & & & 71 \\
Number of respondents & 13 & 20 & 24 & 14 & 67.9 \\
Speech input & $62.5 \%$ & $47.6 \%$ & $66.7 \%$ & $71.4 \%$ & $85.9 \%$ \\
Speech output & $100 \%$ & $70.0 \%$ & $95.8 \%$ & $78.6 \%$ & $79.0 \%$ \\
Ratio & $62.5 \%$ & $68.0 \%$ & $69.6 \%$ & $90.8 \%$ & \\
\hline
\end{tabular}


either male or female respondents or respondents in any country were uninterested in any of the functions listed. Men were generally more interested in all the functions than women, indicating that they might be interested in the robot having more functions than women.

Both men and women and respondents in all the four countries found the appearance of the robot very important, with women considering it slightly more important than men and respondents in the UK giving it the greatest importance and in Italy the least. Men gave their highest evaluation to the obvious robot and women to the humanoid robot, though the differences were not statistically significant and neither men nor women were particularly enthusiastic about their preferred design. There were some differences in preferences for the different designs across the countries. However, other than the French reactions to the obvious robot and, to a lesser extent, the Italian and Polish reactions to the mobility scooter and robotic dog respectively, respondents across all the countries showed a lack of very positive reactions to all the designs.

Respondents in all four countries and both men and women were very strongly interested in using the robot in all the suggest circumstances and differences between the different groups were generally small. Both men and women and respondents in all four countries were interested in using speech for both receiving information and giving instructions to the robot, with greater preferences for using speech to receive information than to give instructions. The order of preferences for receiving information was speech, keypad, joystick or switch for respondents of both genders and in all four countries and only small, though very varied percentages of respondents wanted to receive information in Braille.

\section{Proposed robotic guide: Design using the survey results and the CAT model}

The information obtained from the survey questionnaire and reported in this and the companion paper will now be used to develop design specifications for a mobile robotic guide for blind and visually impaired people. As discussed by the first author [12], there are a number of different ways of organising end-user information and using it to derive design specifications. The Comprehensive Assistive Technology (CAT) model developed by the authors $[13,14]$ will be used here to structure the information and to provide a framework for deriving design specifications. Further work will then be required to develop specific detailed designs from these design specifications, construct mock-ups of the chosen designs and use them to obtain end-user feedback, which can be used to improve the design. A literature overview carried out by the authors and reported briefly in Section 2 will be used in conjunction with the authors' expertise to inform the design in terms of best practice and what is currently technologically feasible.

Due to space limitations only a brief overview, rather than a full discussion of the CAT model will be given and the discussion will focus on the aspects of the design and model for which information was obtained from the survey. The CAT model is a hierarchical structured model, with the four components of the person, the context, the activity/ies, and the (assistive) technology at the top level (see Fig. 2) and subdivisions of these components at the second and third levels.

\subsection{Person}

The survey results showed that there was interest in using the device from both men and women, both blind and visually impaired people, people of a range of ages, with different ages of onset of blindness or visual impairment, and blind or visually impaired people with additional impairments. The interest from respondents in different countries implies that the device could be used throughout Europe, as well as in North America and probably also Japan, Australia and New Zealand. While most of the respondents used mobility aids and travelled effectively with them, there were also respondents who did not go out at night or did not feel particularly confident with their existing mobility aid. The initial design will be aimed at experienced travellers

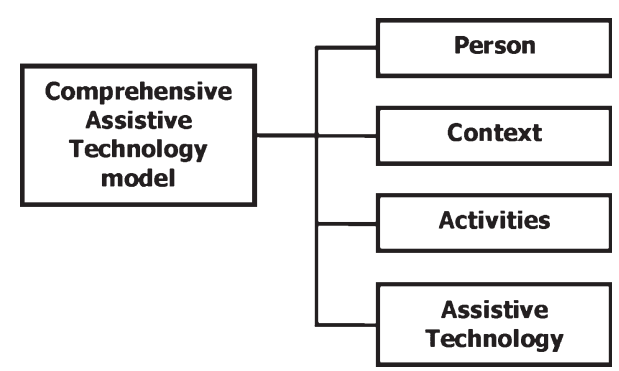

Fig. 2. Comprehensive Assistive Technology (CAT) model. 
over the age of 16 who are using a (long) cane or dog as their main or backup travel aid and have the experience to know when to override the information from the robotic guide, as this is the group who can most safely use the device with the current state of the technology.

Further designs will be aimed at other user groups, including children and young people under 16, older people with physical impairments and the need for physical support and people who only travel accompanied and/or rarely go out, but would be interested in travelling independently and people who do not require a travel aid during the day, but experience significant barriers to independent travel at night. Further research aimed specifically at these user groups would be required to support appropriate design modifications or new designs which take full account of their requirements and preferences.

\subsection{Context and activities}

\subsubsection{National context}

Although this will not be discussed in full, it will be noted that, at present, use of the device will only be feasible in the richer countries with a well-developed modern infrastructure and mechanisms for financing and distributing assistive technology. If it is successful in these countries, it would be hoped to extend its use to other countries and it may be necessary to modify the design to make this feasible.

\subsubsection{Local settings}

Respondents expressed interest in using the robotic guide in a wide range of settings both inside and out, with particular interest in using it for travelling to new destinations, which are likely to be very varied, as well as very significant interest in using it everyday. Settings in which respondents wanted to use the device included complex closed environments, such as railway stations, supermarkets, airports and leisure facilities; campus environments, such as large hospitals, research and academic centres; and complex outdoor environments, such as streets with road works and urban shared spaces. While the device would generally be used on smooth surfaces, there was interest in it being able to move over grass and being able to use it in the countryside, woods and large parks. Respondents were also interested in being able to use the device in all weathers.

\subsubsection{Activities}

Independent travel, including obstacle avoidance, navigation and orientation, using public transport and access to urban and non-urban environments.

\subsection{Assistive technology}

Due to the importance of the Assistive Technology component of the model for the development of design specifications, a complete overview of this component

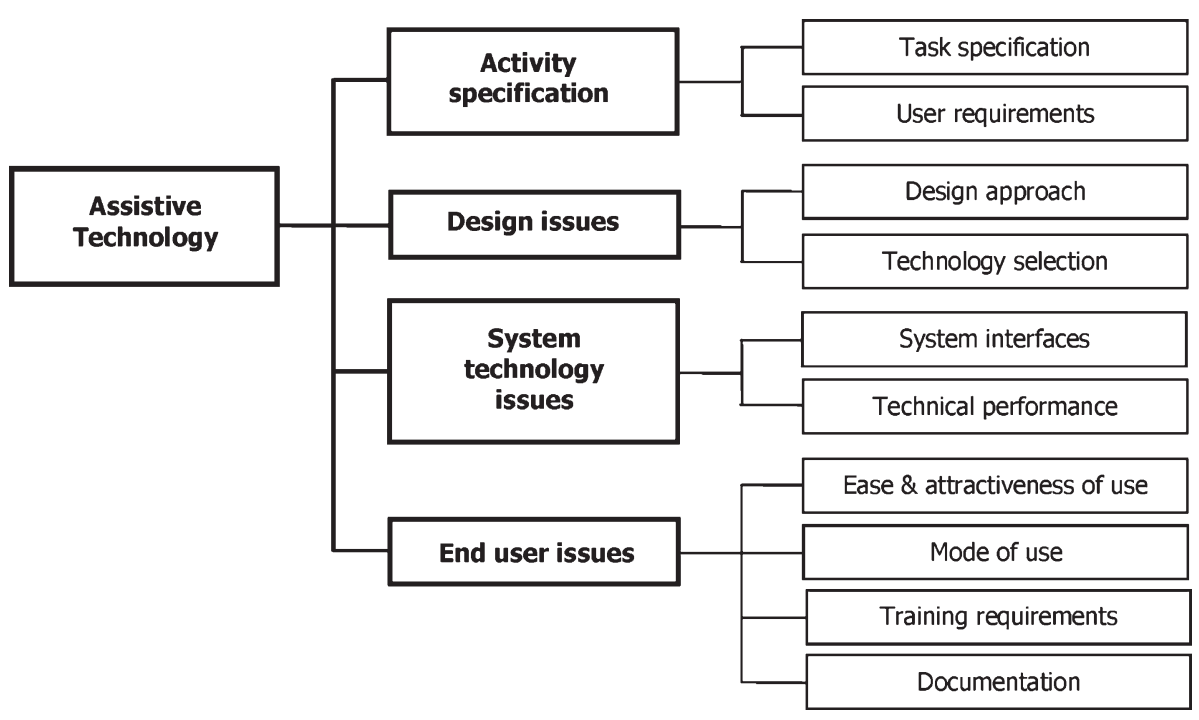

Fig. 3. Model for assistive technology attribute in the CAT model. 
is provided in Fig. 3, with the level of detail that can be considered in a preliminary assistive technology design assessment.

\subsubsection{Task specification}

Respondents expressed an interest in carrying out the following tasks.

- Obstacle avoidance: moving and stationary obstacles of varying types and locations, including at a height.

- Navigation and wayfinding: when travelling on foot and by public transport. Information about the route, points of interest in the vicinity, and the time and distance to the destination, as well as advice on safe routes and routes to avoid.

- Localisation: informing the user of their current location and finding (the entrances of) buildings and other facilities

- Reading information: street names, house numbers and names, building names and announcements, add information boards in or in front of buildings.

- Public transport: finding public transport stops and station entrances, finding and reading the numbers and destinations of public transport vehicles and information boards, as well as providing information on the approaching stop.

- Crossing the road: locating pedestrian crossings and informing the user of the status of traffic lights and when the lights change.

- Emergency: contacting the emergency services and/or the user's family and giving them the user's location when requested

Other facilities of interest to respondents of potential interest to end-users, such as the ability to locate goods or recognise the colour of clothes and other items could be added later. The list of tasks in the task specification will form the basis of a modular software architecture, with one or more modules corresponding to each task, though the earliest versions may not have all the functions in this list. This will facilitate development, the use of existing components and software and adding or removing functions.

\subsubsection{User requirements and issues}

Requirements obtained from the questionnaire responses include the following:

\subsubsection{Appearance, portability and ease of use}

- Appearance: inconspicuous and discreet, but attractive and elegant. Does not draw attention to the user.

- Portability: lightweight and small size, so that it is easily portable and can be put in a pocket or on or under a table and taken on public transport and into the workplace or leisure facilities. The requirement that the robot can be stored on or under a table is more realistic in terms of the various functions required.

- Battery: long life of at least 16 hours and preferably several days; easy to recharge without the use of vision.

- Ease of use: easy and intuitive to use, needs little training and minimal documentation. To be accessible to all users, any documentation should be available electronically, in Braille and as audio on $\mathrm{CD}$, as well as in large and standard size print. It should be presented in clear nontechnical language and designed for use without graphics.

\subsubsection{Reliability, interface, adaptations and ergonomics}

- Robustness and reliability: including to weather, water and knocks and bumps. It should require only minimal maintenance.

- User adaptations: different versions or customisable for different groups of users.

- Accessible interface: to blind and visually impaired people. Most users want high quality synthetic speech output, possibly in combination with vibro-tactile output, but a few users want Braille. Users are interested in speech input, followed by the use of a keypad. A number of users would like a choice of options and the ability to customise the user interface.

- Ergonomics: a long handle will be required to allow the robot to move along the ground, without the user having to bend over to hold it. A telescopic handle will give a compact package when not in use.

\subsubsection{System interface}

The survey results indicate that the robot should have high quality synthetic speech output, as well as vibro-tactile and possibly other outputs, but not Braille. 
Similarly, the inputs should include a combination of speech and a keypad, possibly with additional options. Interface customisation should be investigated. Since speech recognition technology still has a number of limitations, particularly in noisy environments, initially speech input should be limited to a number of specific commands which sound very different from each other. A means, such as pressing a button on the robot handle, of indicating the start and end of speech commands to the robot will be required to avoid confusion with environmental sounds. In addition, obstacle avoidance will be carried out by the robot changing its direction to avoid the obstacle and the change in direction communicated to the user by them sensing the movement through the handle connected to robot. This is the principle of a human or dog guide. It is very intuitive and easy to understand and avoids the problem associated with many travel aids of providing information in a format which is difficult to interpret.

\subsubsection{Design issues \\ 4.3.4.1. Design specifications}

- Stability: stationary and in motion.

- Reliability and robustness: reliable performance, long battery life and a robust design.

- Appearance: small size, lightweight and attractive, but inconspicuous.

- Components: off-the-shelf, readily available components, both to reduce costs and to facilitate repair.

- Environmental considerations: a modular software architecture which facilitates upgrading, good quality recycled materials for the hardware and a design that facilitates component separation for recycling at end-of-life without compromising stability and robustness.

\subsubsection{Design approach}

In line with good practice in user centred design, the design approach will be based on cycles of design, enduser feedback of the design mock-up or prototype and modification. The initial design will draw on the feedback obtained from the survey questionnaire. Since end-users will be blind or visually impaired, any design mock-ups will need to be investigated through touch. Where possible, the design will be based on existing technologies and techniques.

\subsubsection{Technology}

In line with the overview of the literature, the robot will have some combination of ultrasonar and laser sensors, as well as a camera and a global positioning systems (GPS) module, and probably also a radio frequency identification (RFID) reader.

\subsubsection{Software}

- Distributed modular systems architecture: based on task specification. This architecture will facilitate the addition of further functions, including tasks not included in the first version.

- Parallel processing and algorithm optimisation: to reduce processing requirements and ensure that all processing can be carried out on-board the robot.

Possible designs of some of the software modules will be considered briefly below, but space limitations prevent a more detailed discussion or consideration of all the options.

\subsubsection{Robot localisation}

- Effective and accurate localisation: prerequisite for successful navigation and wayfinding.

- Localisation in a wide variety of environments: existing robotic localisation systems are generally restricted to a specific closed indoor environment and rarely consider the effects of moving obstacles such as people. A combination of different technologies will be required.

- Outdoor localisation: two stage localisation with a GPS module to give an initial estimate of the position with 5-20 $\mathrm{m}$ accuracy. This would be followed by second stage localisation using probabilistic and Markov methods based on a combination of grid and topological approaches together with sensor data and open source digital maps where they are available.

- Indoor localisation: the second stage approach could be used, together with input data to generate a digital map if one is not available. In some cases the environment may be marked by RFID tags or other indicators which could be used to provide additional information to support localisation.

\subsubsection{Obstacle detection and avoidance}

Obstacle detection would be provided by the system sensors, for instance ultrasonar and laser sensors and the camera system, in conjunction with software. 
This software could have various functions, including an estimation of the likely future trajectories of moving obstacles, particularly other pedestrians. The robot would use this algorithm to determine whether the current trajectory is likely to intersect or approach the obstacle (trajectory) within a safety margin. If this is the case, an alternative trajectory would be calculated to avoid this obstacle and to return to the original trajectory afterwards.

\subsubsection{Navigation to a destination}

Where the user requires navigation support, they will input the destination details through the user interface. The route could be calculated using the GPS module plus corrections obtained from the robot localisation model for outdoor locations and the robot localisation information in combination with appropriate optimisation and route plotting software for indoor locations. The robot would then follow the route, detouring to avoid obstacles as required, and the user follow the robot, using the information about the robot's movements transmitted through the handle. As with a GPS, the user could be given the option of reviewing the route in advance of travel. The user could also have the option of turning off route following at any point.

\subsubsection{Robot locomotion}

The survey results did not strongly favour either wheeled or legged robots. However, wheeled robots do not have the locomotion and stability problems associated with legged robots. Therefore, the initial locomotion design to be constructed should be wheeled, with the possibility of developing legged designs later, particularly if a larger-scale survey shows a demand for them. While the movement of the different wheels would need to be coordinated, each wheel could have its own controller to facilitate manoeuvring round complex obstacles.

\subsection{Summary design specifications}

The data presented in the previous three sub-sections will now be summarised to give the design specifications below. These include a target initial user group, with later versions to be developed for other user groups. It should be noted that the functionality has been divided into functions to be included in the initial version and functions to be added later. Although there is an element of arbitrariness in this division, it does take account of what is currently feasible technologically without requiring additional infrastructure, such as additional RFID tags or infrared beacons, to be installed in the environment.

The design specifications are as follows

\subsubsection{End-users, appearance and functionality}

- (Initial) target end-user group: blind and visually people of both sexes over the age of 16 who are experienced travellers and come from countries, such as those in Europe, Northern America and Japan, with a well-developed infrastructure. They should be open to using new technologies and not have significant additional impairments.

- Appearance: small size, light weight, compact, attractive, but inconspicuous, telescopic handle, wheeled locomotion.

- Functionality: initial functionality to include obstacle avoidance; navigation to destination; indoor and outdoor localisation information about the route and points of interest; locating public transport stops and stations and pedestrian crossings. Functionality to be added later: reading street names and numbers, bus and train numbers and information boards; contacting emergency services and user's family when required; status of traffic lights.

- Other specifications: robust, including to weather and knocks, and reliable; low maintenance; easy and intuitive to use; minimal training and documentation required; all documentation in simple language and available in alternative formats.

\subsubsection{Hardware, software and system interface}

- Software design: distributed modular architecture based on task specifications/system functions to which additional functions can be added later; parallel processing and system optimisation.

- Sensors: combination of laser and ultrasonar sensors, probably also camera, GPS and an RFID reader.

- Hardware: long life battery, on-board processing.

- System interface: high quality synthetic speech and vibro-tactile output and keypad and speech input, with a limited number of specific speech commands and an indication of the start and end of the speech input. Trajectory changes to avoid obstacles sensed through the handle. 


\section{Discussion and conclusions}

Part 1 of this paper presented the methodology and an overview, analysis and discussion of the results of a survey to investigate the attitudes, requirements and preferences of blind and visually impaired people for a robotic guide. The current Part 2 presents the gender and country based analysis of the data and uses the responses to derive design specifications for a robotic guide.

It should be noted that this is one of the first multilanguage cross-country surveys of end-user attitudes, preferences and requirements for assistive technology to be carried out. The country based analysis involved France, Italy, Poland and the UK, as the countries with the largest numbers of respondents. Data was also obtained from Belgium, Canada, Spain and the United States, but there were insufficient responses to analyse this data separately. Data obtained from England, Northern Ireland and Scotland was also all considered as UK data for the same reason.

There was considerably agreement across countries and gender on the functions the robot should have and the circumstances in which it would be useful. While there was a high level of demand for most of the suggested functions, women were generally less interested in each of the functions than men and may have wanted the robot to have fewer functions overall. There was also general agreement on the importance of the robot's appearance and a general lack of enthusiasm for all the proposed designs, other than the French evaluation of the obvious robot and, to a lesser extent, the Italian and Polish evaluations of the mobility scooter and robotic dog respectively. However, though there was a reasonable degree of agreement across gender lines on the preferences for the different designs, there were considerable differences in preferences in the different countries. While speech was universally preferred for both input and output and there was general agreement on the order of preference for system input, there were differences in the percentages of respondents interested in the different options across both gender and countries

Despite differences on some of the details, the results of the gender and country based analysis of the survey show considerable consensus on the attitudes, requirements and preferences across country and gender. This makes it feasible to develop a single version of the robotic guide which will be attractive to both women and men and in a number of differ- ent countries. However, subsequent development could examine customisation to take account of some of the small differences in preferences.

The results of the survey presented in this paper and Part 1 were then applied to obtain preliminary design specifications for a robotic guide. The Comprehensive Assistive Technology (CAT) model was used to organise the information and structure the discussion. A summary of the design specifications obtained was presented in Section 4.4 and will not be repeated here. When the development of the robotic guide proceeds, the next stage would be the construction of a number of design mock-ups based on the design specifications for testing with end-users. Since end-users are blind and visually impaired people, these mock-ups should be designed to allow investigation of all their relevant features through touch. The investigation of the mockups will result in the choice of one of more designs for development, possibly after iterative cycles of modification of the design specifications and modification of the existing design mock-ups or construction of new ones.

\section{Acknowledgments}

We would like to thank the Leverhulme Trust for the provision of a Research Fellowship to Dr Hersh out of which this work developed, all the blind and visually impaired people who completed questionnaires and circulated them for me, as well as Hanna Pasterny, Marie-Christine Amato, Daniele Rigoldi and Abdeleh Akel for help in improving the French, Italian and Polish versions of the questionnaire.

\section{References}

[1] J. Borenstein and Y. Koren, The vector field histogram - fast obstacle avoidance for mobile robots, IEEE J. Robotics and Automation 7(3) (1991), 278-288.

[2] U. Borgolte, H. Hoyer, C. Bühler and H. Heck, Architectural concepts of a semi-autonomous wheelchair, J. Intelligence and Robotic Systems 22 (1998), 233-253.

[3] W. Burgard, A.B. Cremers, D. Fox, H. Dähnel, G. Lakemeyer, S. Schulz, W. Steiner and S. Thrun, Experiences with an interactive museum tour-guide robot, Artificial Intelligence, 114(1-2) (1999), 3-55.

[4] A. Clodic et al., Rackham: an interactive robot-guide, 15th IEEE Symp, On Robot and Human Interactive Communication, (2006), 502-509.

[5] O. Diegel, A. Badve, G. Bright, J. Potgieter and S. Tlale, Improved mecanum wheel design for omni-directional robots. 
Proc. 2002 Conf Robotics and Automation, Aukland, 2002, 112-121.

[6] A. Elfes, Sonar-based real-world mapping and navigation, IEEE J Robotics and Automation 3(3) (1987), 249-265.

[7] D. Fox, W. Burgard and S. Thrun, The dynamic window approach to collision avoidance, IEEE Robotics and Automation Magazine, (1997), 23-33.

[8] C.P. Gharpure and V.A. Kulyukin, Robot-assisted shopping for the blind: issues in spatial cognition and product selection, Intel Service Robotics 1 (2008), 237-251.

[9] D. Golubovic and H. Hu, A hybrid evolutionary algorithm for gait generation of Sony legged robots. 28th Ann. Conf, IEEE Ind Electronics Soc, Sevilla, Spain, 2002.

[10] P. Gregorio, M. Ahmadi and M. Buehler, Design, control and energetics of an electrically actuated legged robot, IEEE Trans Systems, Man and Cybernetics Part B 27(4) (1997), 626-633.

[11] M.A. Hersh The application of information and other technologies to improve the mobility of blind, visually impaired and deafblind people, Travel Health Informatics and Telehealth, Selected Papers from EFMI Special Topic Conference, Antalya, Turkey, G, Mihalaş et al. (eds.) pp. 11-24, Victor Babes University Publishing House, 2009.

[12] M.A. Hersh, The design and evaluation of assistive technology products and devices part 1, design, International Encyclopedia of Rehabilitation, 2010. http://cirrie.buffalo.edu/ encyclopedia/en/article/309/

[13] M.A. Hersh and M.A. Johnson, On modelling assistive technology systems part I: modelling framework, Technology and Disability, 20(3) (2008a), 193-215.

[14] M.A. Hersh and M.A. Johnson, On modelling assistive technology systems part II: Applications of the comprehensive assistive technology model, Technology and Disability, 20(4) (2008b), 251-270.

[15] M.A. Hersh and M.A. Johnson, A robotic guide for blind people part 1: a multi-national survey of the attitudes, requirements and preferences of potential end-users, Applied Bionics and Biomechanics 7(4) (2010), 277-288.

[16] K. Hirai, M. Hirose, Y. Haikawa and T. Takenaka, The development of Honda humanoid robot. Proc 1998 IEEE Int Conf Robotics and Automation, Leuven, Belgium, 1998, 1321-1325.

[17] D. Katić and M. Vukobratović, Survey of intelligent control techniques for humanoid robots, $J$ Intelligent and Robotic Systems 37 (2003), 117-141.

[18] T.W. Kirkman, Statistics to use, 1996. http://www.physics. csbsju.edu/stats/contingency_NROW_NCOLUMN_form. html. Accessed 6-10.1.10.
[19] D. Kortenkamp and T. Weymouth, Topological mapping for mobile robots using a combination of sonar and vision sensing, Proc 12th Nat Conf AI. Menlo Park, AAAI Press/MIT Press, 1994, 979-984.

[20] B. Kuipers and Y-T. Byun, A robust qualitative method for spatial learning in unknown environments, Proc 8th Nat Conf AI AAAI-99, Menlo Park, Cambridge, AAAI Press/MIT Press, 1988.

[21] Ø. Langsrud, undated. http://www.langsrud.com/fisher.htm. cited 6-10.1.10

[22] H.P. Moravec, Sensor fusion in certainty grids for mobile robots, AI Magazine Summer (1988), 61-74.

[23] E. Prassler, J. Scholz and P. Fiorini, Navigating a robotic wheelchair in a railway station during rush-hour, Int $J$ Robotics Research (1999).

[24] E. Prassler, J. Scholz and P. Fiorini P., A robotic wheelchair for crowded public environments, IEEE Robotics and Automation Magazine, (2001), 38-45.

[25] R.C. Simpson, S.P. Levine, D.A. Bell, L.A. Jaros, Y. Koren and J. Borenstein, Navchair: an assistive wheelchair navigation system with automatic adaptation, In Mittai VA et al. (eds.), Assistive Technology and AI, Berlin, Springer Verlag (1998), 235-255.

[26] S. Thrun, M. Beetz, M. Bennewitz, W. Burgard, A.B. Cremers, F. Dellaert, D. Fox, D. Hähnel, C. Rosenberg, N. Roy, J. Schulte and D. Schulz, Probabilistic algorithms and the interactive museum tour-guide robot Minerva, Int J Robotics Research 19(11) (2000), 972-999.

[27] C. Urdiales, A. Bandera, E. Pŕerez, A. Poncela, F. Sandoval and Sandoval, Hierarchical planning in a mobile robot for map learning and navigation. In: Zhou C et al. (eds.), Autonomous robotic systems:soft computing and hard computing methodologies and applications, Heidelberg, Physica Verlag $\mathrm{GmbH}$, 2003, 165-188.

[28] H. Uustal and J. Minkel J., Study of the independence IBOT 3000 mobility system: an innovative power mobility device, during use in community environments, Archives of Physical Medicine and Rehabilitation, 85(12) (2004), 2002-2010.

[29] T. Zielinska and J. Heng, Development of walking machine: mechanical design and control problems, Mechatronics, 12 (2002), pp.737-754.

[30] C. Zhou and K.H. Low, Combined use of ground learning model and active compliance to the motion control of walking robotic legs, Proc 2001 IEEE Internat Conf Robotics and Automation, Seoul Korea, 2001, 3159-3164. 

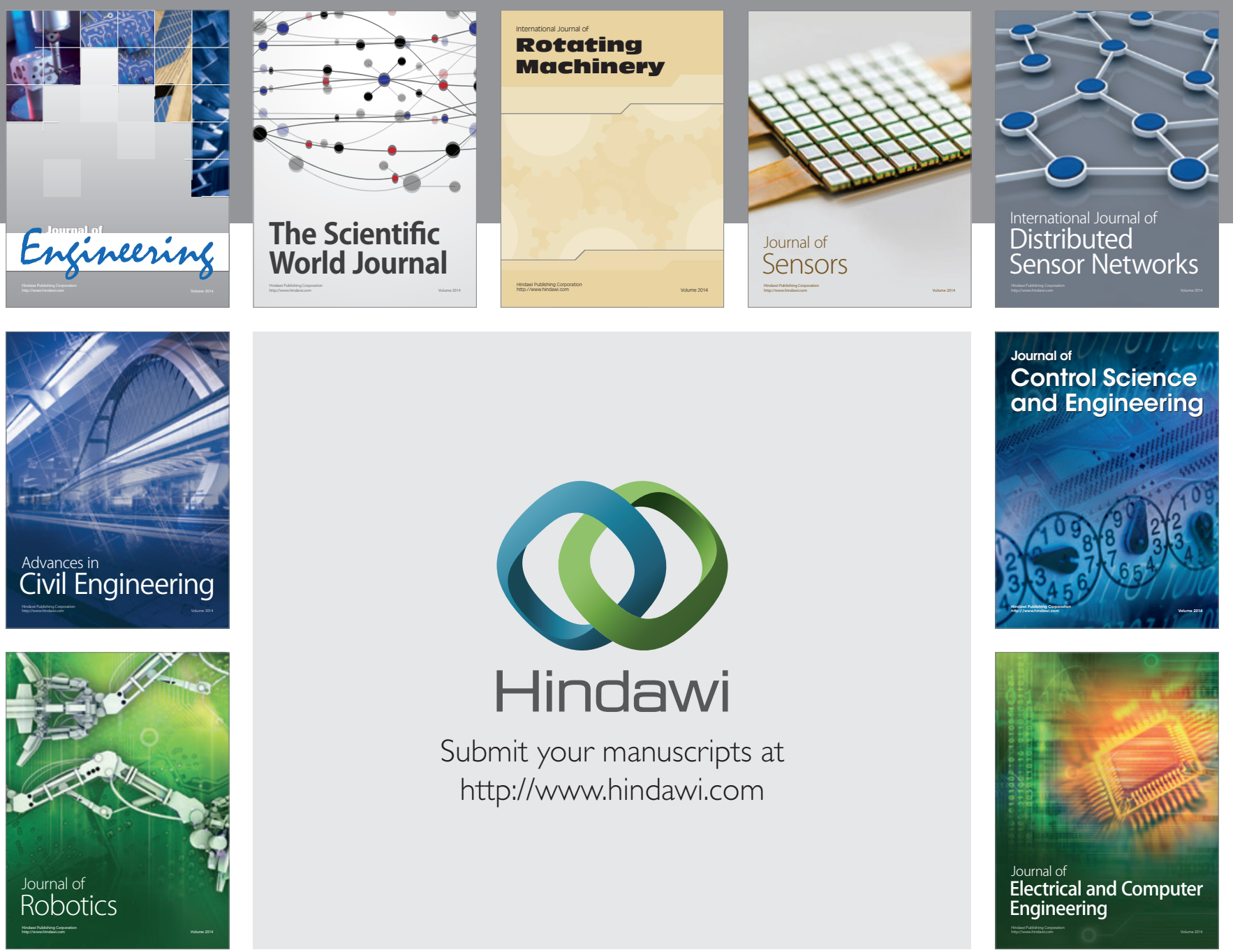

Submit your manuscripts at

http://www.hindawi.com
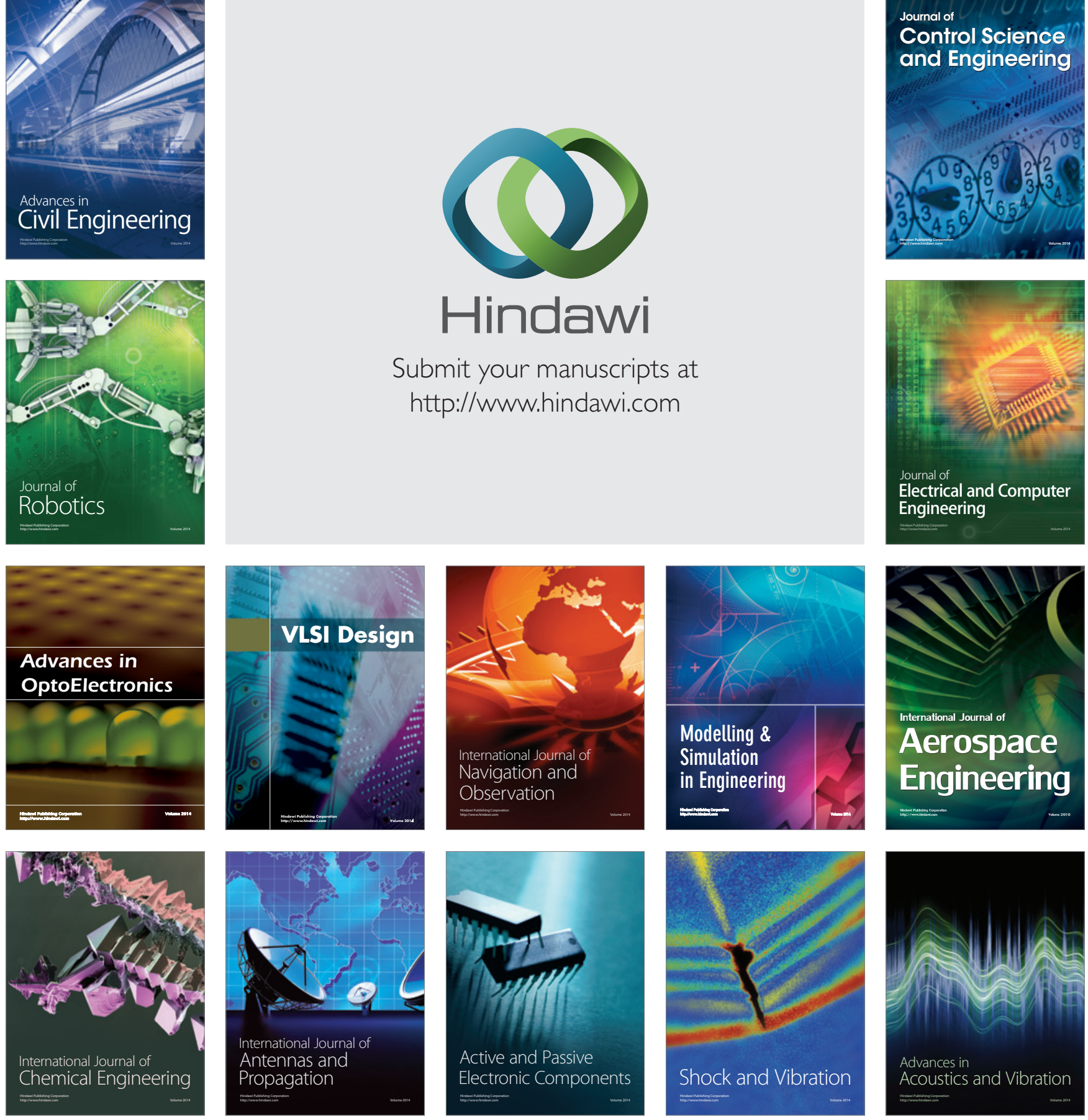